\title{
¿Existen diferencias en las características y en el comportamiento del tumor vesical según edad de presentación? Nuestra experiencia
}

\author{
Serrano Frago P, Gil Martínez P, Gil Sanz MJ, Felipo F*, Allué López M, Rioja Sanz LA. \\ Servicio Urología. *Servicio Anatomía Patológica. Hospital Universitario Miguel Servet. Zaragoza.
}

Actas Urol Esp. 2007;31(8):825-830

\section{RESUMEN}

¿EXISTEN DIFERENCIAS EN LAS CARACTERÍSTICAS Y EN EL COMPORTAMIENTO DEL TUMOR VESICAL SEGÚN EDAD DE PRESENTACIÓN? NUESTRA EXPERIENCIA

Objetivo: Dados los diferentes resultados de las series que evalúan el comportamiento de los tumores vesicales según la edad en la que debutan, nuestro objetivo se basa en valorar las características y comportamiento según edad de aparición.

Método: Se realiza un estudio retrospectivo de los tumores vesicales de nuevo diagnóstico de nuestra área durante el decenio 1993-2003, distribuidos en 3 intervalos etarios y se evalúan diferentes características y comportamiento.

Resultados: Se demuestra que los pacientes de más edad presentan tumores más grandes, indiferenciados y con mayor tasa de progresión hacia infiltrantes. Además aparecen como factores significativos independientes en el estudio multivariante: la edad, el estadio patológico y el grado tumoral.

Conclusiones: En nuestra experiencia, los pacientes de edad mayor de 70 años presentan neoplasias de características clínicas similares, aunque patológicamente más agresivas, con mayor porcentaje de progresión y peor supervivencia.

Palabras clave: Tumor vesical. Edad. Comportamiento.

\section{ABSTRACT}

ARE THERE ANY DIFFERENCES BETWEEN THE CHARACTERISTICS AND THE BEHAVIOR OF BLADDER CANCER ACCORDING TO THE AGE OF PRESENTATION? OUR EXPERIENCE

Introduction: Owing to the different results from the series that evaluate the behavior of the bladder cancer according to the age at the moment of the diagnosis, our objective is based on valuing the characteristics and behaviour according to age of appearance.

Methods: A retrospective study of bladder cancer diagnosed in our area during decade 1993-2003, distributed in 3 intervals of age and some characteristics and behaviour are valued.

Results: Elderly patients present greater tumors, non differentiated and with greater rate of progression to infiltrated. Moreover the age, the pathological stage and the tumorlike degree appear as independent significant factors in the multivariant study.

Conclusions: In our experience, the patients greater than 70 years present neoplasms of similar clinical characteristics, although pathologically more aggressive, with greater percentage of progression and worse survival.

Keywords: Bladder cancer. Age. Behaviour.

$\mathrm{E}^{1}$ cáncer de vejiga ha representado casi un $6 \%$ de las defunciones por cáncer en hombres y un $2 \%$ en mujeres durante el año 2000. Supone la $5^{\text {a }}$ causa de muerte por cáncer en hombres y la $13^{a}$ en mujeres en España, siendo la razón de sexos de $4: 1^{1}$.
En relación a la incidencia según sexos se han publicado diversos estudios en diferentes regiones españolas y uno de ellos realizado en Madrid en 1994 por Arrizabalaga M. et al. exponía una relación de 4 varones/ 1 mujer, en comparación a otros con mayor relación pero no existen datos globales del país ${ }^{2}$. 
El tumor vesical afecta a personas en la sexta década de la vida aunque puede aparecer a cualquier edad, la edad media de presentación 69 años en el varón y 81 años en la mujer ${ }^{3}$.

Se ha expuesto en diversos estudios la menor agresividad del tumor vesical por comportamiento biológico mejor según la edad de presentación, con diferentes puntos de cortes de edad de referencia para pacientes jóvenes; sin embargo no se demuestra en todas los trabajos presentados de diferentes países ${ }^{4}$.

\section{OBJETIVO}

Ante la existencia de diferentes series y resultados contradictorios en lo referente al distinto comportamiento de los tumores vesicales según la edad en la que se diagnostica por primera vez el tumor vesical, nuestro objetivo es valorar qué ocurre con nuestros pacientes.

El estudio nace a partir de los tumores tratados en nuestro servicio durante un periodo de tiempo y valorar las características y comportamiento del mismo según una categorización realizada por la edad en la que debuta el tumor vesical.

\section{MATERIAL Y METODOS}

Se realiza un estudio retrospectivo de todos los tumores vesicales que han sido diagnosticados por primera vez durante el periodo de tiempo 1993-2003, con un global de 1.002 pacientes de nuestra área sanitaria correspondiente. Tomando como referencia la edad en la que se diagnosticó y trató por primera vez el tumor vesical se realiza una división de los mismos en tres grupos: menores de 50 años, de 50 a 70 años y mayores de 70 años. A estos pacientes se realizó un estudio de las características clínicas y patológicas del tumor vesical durante una mediana de seguimiento de 48,9 meses (IQ: 54,7).

De las historias clínicas se obtuvieron datos de sexo del paciente, presencia en algún momento de su vida de hábito tabáquico según respuesta del propio paciente en la anamnesis así como de consumo habitual de alcohol. De los datos administrativos se obtuvo el medio de vivienda ya fuera en núcleo urbano o rural. Se registró la presentación clínica del tumor vesical (macrohematuria, microhematuria, sintomatología del tracto urinario inferior, hallazgo incidental mediante pruebas diagnosticas como ecografía o tomografía, y hallazgo casual durante otra cirugía). Se reflejó el método por el que se diagnóstico el tumor (ecografía, cistoscopia, urografia, tomografía, revisión endoscópica o pieza de anatomía por otro motivo) y el tiempo que transcurrió entre la presentación clínica y la realización de la resección transuretral del mismo en meses.

Revisando los datos anatomo-patológicos del registro del Servicio de Anatomía Patológica de nuestro hospital se registraron datos como el estadio patológico inicial según la clasificación internacional contra el cáncer TNM $6^{\text {a }}$ edición del año 2002 agrupados en superficiales (Tis, Ta o T1) e infiltrantes (T2,T3 y T4); el grado tumoral según clasificación publicada por la Organización Mundial de la Salud en 1973, la presencia de más de un tumor vesical y características como el diámetro en centímetros y la positividad ( presente en mas del 20\% de las células de la muestra) o no de presencia de proteína p53 y ki67.

En los tumores vesicales que debutan en estadio Tis, Ta o T1 se clasifican por grupos de riesgo de recidiva y progresión (según guías clínicas de la Asociación Europea de Urología del 2001) en alto, medio y bajo riesgo.

Además durante el periodo de seguimiento de los mismos se evaluó aquellos que debutando como tumor vesical considerado superficial (Tis, Ta o $\mathrm{T} 1$ ) en alguna recidiva posterior se convierte en tumor infiltrante (T2, T3 ó T4) y el tiempo que trascurre hasta que esto ocurre en meses.

Se valoró los pacientes que fallecían por causas secundarias al tumor vesical, aquellos que lo hacían por otras causas y los que permanecían vivos.

Con estos datos registrados, mediante el programa estadístico SPSS 13.0 se realizó un estudio univariante de cada uno de ellos según los tres grupos de edad descritos. Así con aquellas que presentaban diferencias estadísticamente significativas se realizó un estudio multivariante de supervivencia cáncer específico mediante regresión de Cox.

\section{RESULTADOS}

La media de edad de los pacientes diagnosticados de tumor vesical era de 68 años. De los que 
el grupo con más número era el de 50-70 años con un $47,1 \%$ (470 pacientes), muy seguido por el grupo de más edad con 45,7\% (457 pacientes) y por último los más jóvenes representando el $7,2 \%$ (75 pacientes).

Dentro de las características clínicas estudiadas en los grupos etarios según edad del diagnostico inicial, el sexo predominante es el del varón, $88 \%$ son varones en el grupo de 50-70, $81 \%$ en el de más de 70 y $80 \%$ en el de menos de 50 (Chi-cuadrado, $\mathrm{p}<0,011$ ). En cuanto a la presencia en algún momento de su vida del hábito tabáquico, se ponía de manifiesto el mayor porcentaje, con $36 \%$ en los mayores de 70 años, frente al $10 \%$ en los de menos de 50 . (Chi-cuadrado, $\mathrm{p}<0,000$ ) (Fig. 1).

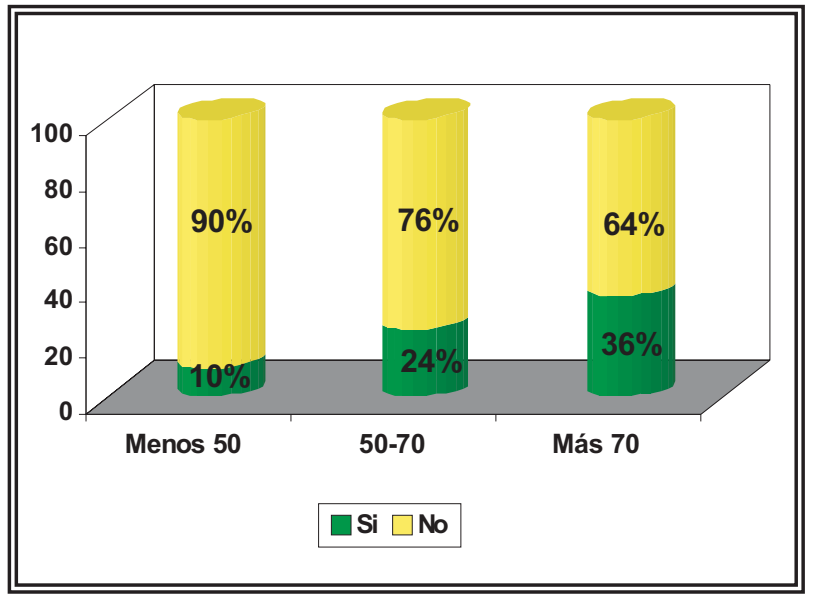

FIGURA 1. Tabaco según grupos.
En el resto de variables clínicas no se observaban diferencias estadísticamente significativas como se muestra en la Tabla 1.

En la valoración de las características patológicas que presentaban los tumores según los grupos de edad, se observó que el grado tumoral indiferenciado era en un porcentaje mayor en los de edad avanzada, y por el contrario, el mayor porcentaje de bien diferenciados aparecía en el grupo de menos de 50 años (Chi-2 p<0,001) como se observa en el siguiente gráfico (Fig. 2).

En el diámetro mayor del tumor de los tumores había diferencias, $2,5 \mathrm{~cm}$. en los mayores de $70,2 \mathrm{~cm}$. en los de 50-70 y $1,5 \mathrm{~cm}$. en los menos de 50. (Kruskal Wallis $\mathrm{p}<0,001$ ).

La distribución según los grupos de riesgo de progresión y recidiva tumoral de la EAU en los tres grupos demostraba ser los de alto riesgo los predominantes en el grupo de mas de 70 años y los de bajo riesgo los predominantes en los menores de 50 años (Chi-2 p=0,019) (Fig. 3).

El resto de variables patológicas estudiadas no presentaban diferencias estadísticamente significativas, como se observa en la Tabla 2.

En el estudio de la progresión de aquellos tumores que debutaban como superficiales hacia infiltrantes en posteriores recidivas tumorales, los pacientes de más edad presentaban un porcentaje 2 veces superior al de edades medias y tres veces superior al de los jóvenes con diferencias estadísticamente significativas (Pearson ChiSquare, $p=0,004$ ) (Fig. 4).

Tabla 1

\begin{tabular}{|c|c|c|c|c|c|}
\hline \multicolumn{2}{|l|}{ Variable } & \multirow{2}{*}{$\begin{array}{c}\begin{array}{c}\text { Menos 50 } \\
\text { (N:75) }\end{array} \\
14 \%\end{array}$} & \multirow{2}{*}{$\begin{array}{c}\begin{array}{c}\mathbf{5 0 - 7 0} \\
\text { (N:470) }\end{array} \\
21 \%\end{array}$} & \multirow{2}{*}{$\begin{array}{c}\begin{array}{c}\text { Mas de } 70 \\
\text { (N: 457) }\end{array} \\
20 \%\end{array}$} & \multirow{2}{*}{$\begin{array}{c}\text { Valor } \mathbf{p} \\
>0,05\end{array}$} \\
\hline Alcohol & $\mathrm{Si}$ & & & & \\
\hline & No & $86 \%$ & $79 \%$ & $80 \%$ & \\
\hline \multirow[t]{2}{*}{ Medio vivienda } & Urbano & $94 \%$ & $90 \%$ & $88 \%$ & $>0,05$ \\
\hline & Rural & $6 \%$ & $10 \%$ & $12 \%$ & \\
\hline \multirow[t]{5}{*}{ Presentación clínica } & Macrohematuria & $78 \%$ & $78 \%$ & $81 \%$ & $>0,05$ \\
\hline & Microhematuria & $5 \%$ & $4 \%$ & $2 \%$ & \\
\hline & Cirugía prostat. & - & $3 \%$ & $3 \%$ & \\
\hline & Incidental & $14 \%$ & $11 \%$ & $11 \%$ & \\
\hline & STUI & $3 \%$ & $7 \%$ & $5 \%$ & \\
\hline \multicolumn{2}{|c|}{ Tiempo desde clínica hasta RTU } & $\begin{array}{c}5,9 \text { meses } \\
\text { (IQ:5,2) }\end{array}$ & $\begin{array}{c}8 \text { meses } \\
(\mathrm{IQ}: 4,5)\end{array}$ & $\begin{array}{l}8 \text { meses } \\
(\mathrm{IQ}: 4,4)\end{array}$ & Kruskal Wallis Test \\
\hline \multirow[t]{2}{*}{ Cistectomías } & $\mathrm{Si}$ & $11 \%$ & $20 \%$ & $9 \%$ & $>0,05$ \\
\hline & No & $89 \%$ & $84 \%$ & $91 \%$ & \\
\hline
\end{tabular}




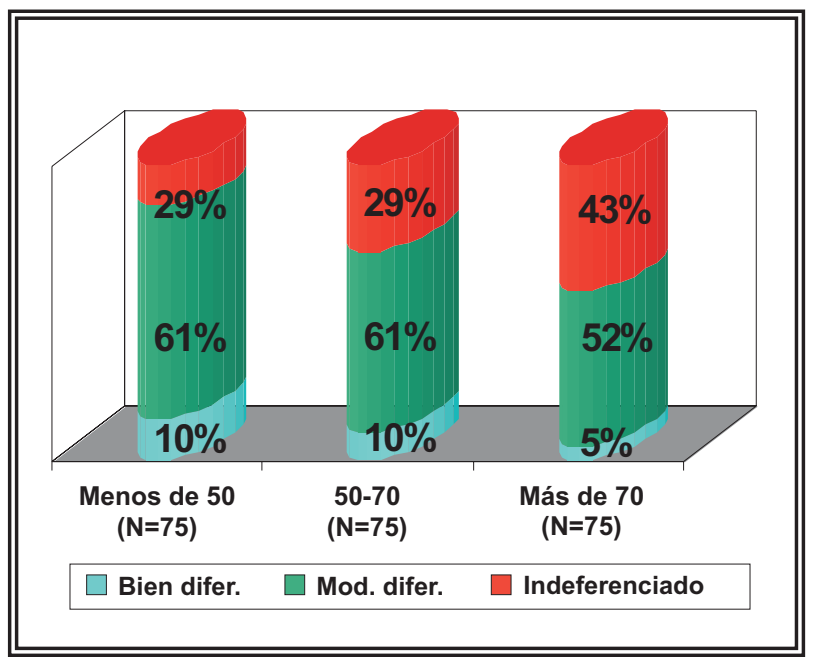

FIGURA 2. Grado tumoral según grupos.

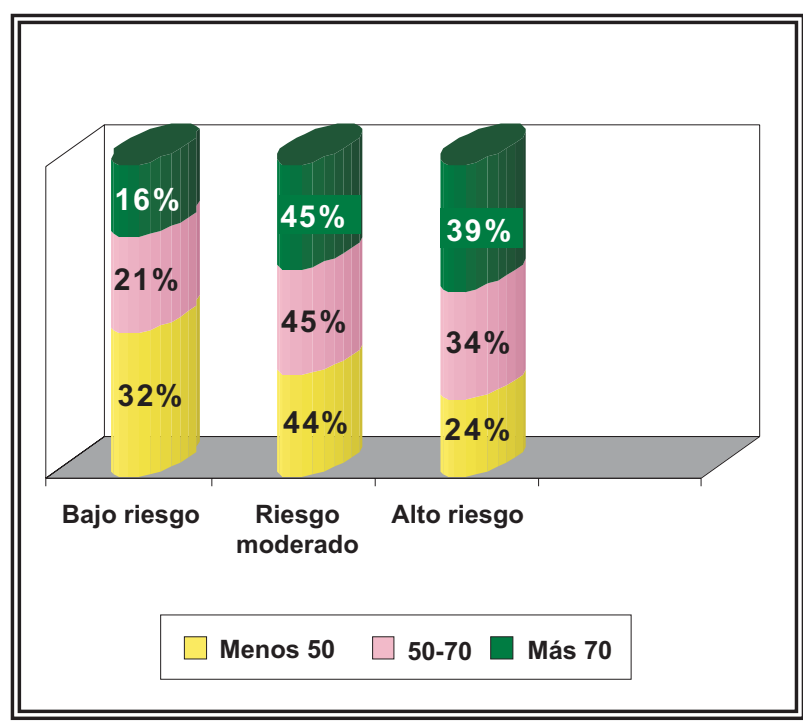

FIGURA 3. Grupos EAU según grupos edad.

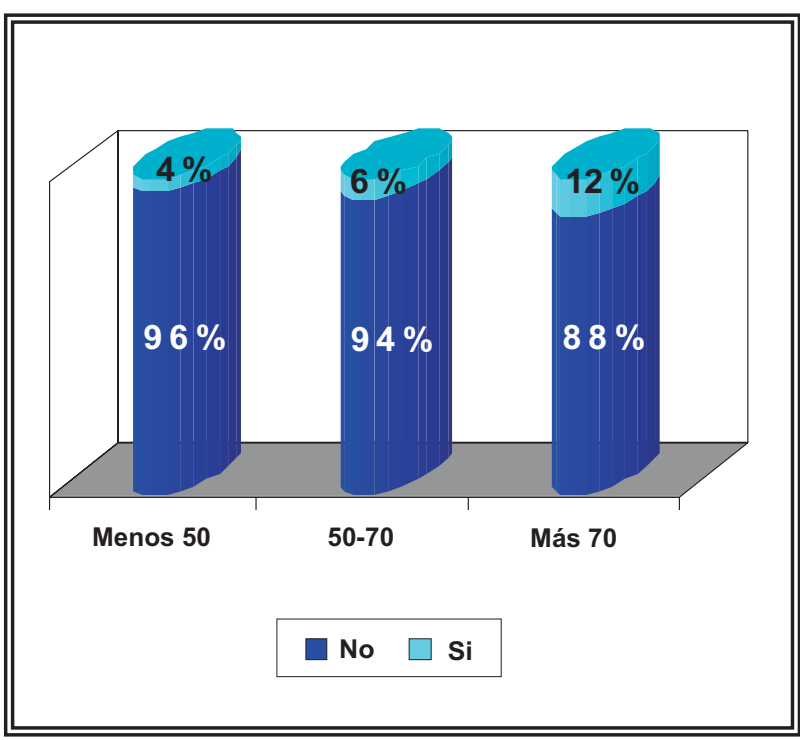

FIGURA 4. Grupos EAU según grupos edad.

De los pacientes que en recidivas posteriores habían progresado a infiltrantes, los de menos de 50 años ocurría en una mediana de 5,9 meses (IQ: 5,2) frente a 8 meses de los de 50-70 (IQ: 4,5) y mas de 70 años (IQ: 4,4) con un valor p:0,000 (Kruskal-Wallis).

En cuanto a la supervivencia cáncer especifica, no se evidenciaban fallecimientos por el tumor vesical en el grupo de menos de 50 años, el grupo de 50-70 tenían una media de 137 meses (SE:1,9) y los de más de 70 años de 99 meses (SE:3,3) (Log Rank Test, p=0,000) (Fig. 5).

En el estudio multivariante realizado con la introducción de las covariables con significación estadística en el estudio arriba presentado, tanto la edad, estadio y grado conservaban influencia significativa en la supervivencia cáncer específica

Tabla 2

\begin{tabular}{|c|c|c|c|c|c|}
\hline Variable & & $\begin{array}{c}\text { Menos } 50 \\
\text { (N:75) }\end{array}$ & $\begin{array}{c}50-70 \\
(\mathrm{~N}: 470)\end{array}$ & $\begin{array}{c}\text { Mas de } 70 \\
\text { (N: 457) }\end{array}$ & Valor $\mathbf{p}$ \\
\hline \multirow[t]{2}{*}{ Estadio patológico } & Superficial & $98,7 \%$ & $98 \%$ & $96,7 \%$ & 0,34 \\
\hline & Infiltrante & $1,3 \%$ & $2 \%$ & $3,3 \%$ & \\
\hline \multirow[t]{2}{*}{ Multifocalidad } & $\mathrm{Si}$ & $40 \%$ & $60 \%$ & $63,6 \%$ & 0,154 \\
\hline & No & $60 \%$ & $40 \%$ & $36,4 \%$ & \\
\hline \multirow[t]{2}{*}{ P53 } & $\mathrm{Si}$ & $35,9 \%$ & $34,5 \%$ & $45,7 \%$ & 0,061 \\
\hline & No & $64,1 \%$ & $65,5 \%$ & $54,3 \%$ & \\
\hline \multirow[t]{2}{*}{ Ki67 } & $\mathrm{Si}$ & $50 \%$ & $50 \%$ & $84,6 \%$ & 0,117 \\
\hline & No & $50 \%$ & $50 \%$ & $15,4 \%$ & \\
\hline
\end{tabular}

Así, se observa que cada año que asciende en el momento del diagnóstico del tumor vesical se multiplica el riesgo de fallecer por 1,086 . Aquellos que presentan grado tumoral G3 tienen 7 veces más riesgo de fallecer por el tumor que en los otros grados tumorales, al igual que los tumores que debutan como infiltrantes respecto a los superficiales. 


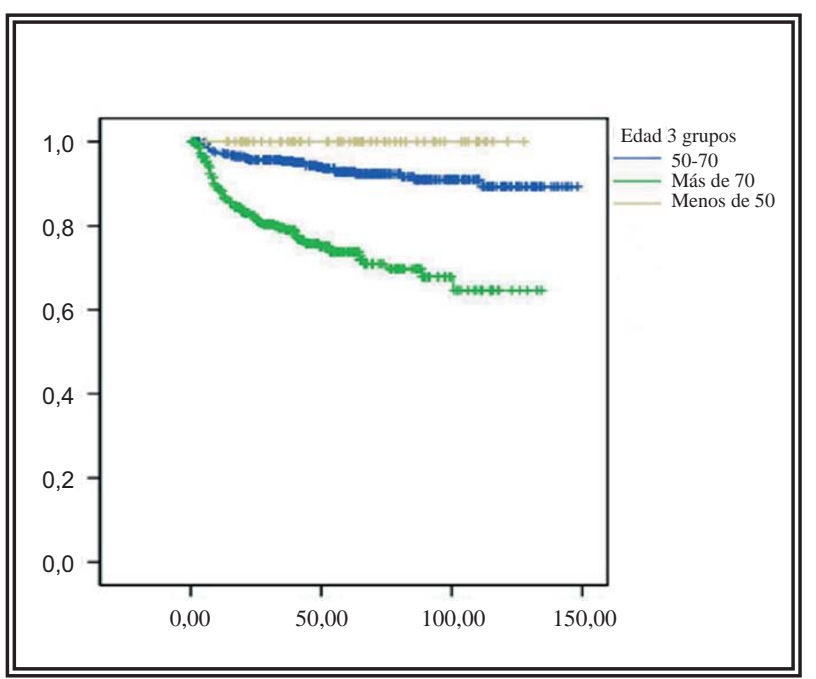

FIGURA 5. Supervivencia cáncer específica.

\section{DISCUSIŌN}

La edad más frecuente de aparición del tumor vesical es en la sexta década de la vida ${ }^{3}$ con una mediana de edad en el momento del diagnóstico que oscila en diferentes estudios entre 68-72 años $^{4-6}$, similar a nuestra serie.

Es dificil plantear la edad de referencia para pacientes jóvenes y mayores, encontrándose con mayor frecuencia el corte en 40 o en los 50 años y 70 en ésta última cuestionándose el tratamiento quirúrgico ${ }^{7}$.

Nosotros realizamos el trabajo estratificando a los pacientes en tres grupos de edad para así poder comparar paciente jóvenes, de edad intermedia y mayores.

Si se realiza una revisión de la literatura se evidencia una discrepancia en cuanto a los resulta- dos de las series que valoran el comportamiento del tumor vesical según la edad de aparición. La mayor parte de los trabajos concluyen que los pacientes en los que aparecen jóvenes presentan mejor comportamiento ${ }^{4,8,9}$. Frente a estos, en menos trabajos no se objetivan diferencias según la edad ${ }^{10}$, incluso peor evolución ${ }^{11}$. En nuestra serie se observa que los pacientes que debutan con tumor vesical por debajo de los 50 años presentan características clínico-patológicas más favorables (tumores mas diferenciados, de menor tamaño, mejores estadios tumorales) y clasificados según grupos de riesgo de recidiva y progresión tumoral según EAU, un mayor porcentaje de bajo riesgo. La progresión de tumor vesical superficial hacia estadio infiltrante ocurría 3 veces menos que en los de mayor edad, y no se objetivaban muertes por el tumor en los más jóvenes.

En el estudio se comporta como factor pronóstico la edad, como se demuestra en el estudio multivariante, siendo junto con el estadio y el grado los que influyen significativamente en la supervivencia cáncer especifica, factores demostrados en múltiples estudios de recidiva y progresión ${ }^{4}$, y no demostrada la importancia de la edad en otros de supervivencia ${ }^{12}$.

\section{CONCLUSIONES}

En los pacientes mayores de 70 años apreciamos neoplasias vesicales más agresivas, con mayor tendencia a progresión y peor supervivencia.

Se demuestra en nuestra serie la influencia de la edad al diagnostico, junto con otros factores ya conocidos (estadio y grado tumoral) en la supervivencia.

Tabla 3. Resultado del análisis multivariante.

\begin{tabular}{|c|c|c|c|c|c|c|c|}
\hline Pasos & Variable & B & SE & Wald & df & Sig. & $\operatorname{Exp}(B)$ \\
\hline \multirow[t]{2}{*}{1} & Infiltr & & & 156,835 & 1 (a) & ,000 & \\
\hline & Infiltr (1) & 2,610 & ,208 & 156,835 & 1 & ,000 & 13,606 \\
\hline \multirow[t]{3}{*}{2} & edad & ,089 & ,012 & 58,505 & 1 & ,000 & 1,093 \\
\hline & Infiltr & & & 158,417 & 1 (a) & ,000 & \\
\hline & Infiltr (1) & 2,657 & ,211 & 158,417 & 1 & ,000 & 14,259 \\
\hline \multirow[t]{6}{*}{3} & edad & ,082 & ,012 & 49,130 & 1 & ,000 & 1,086 \\
\hline & Infiltr & & & 69,385 & 1 (a) & ,000 & \\
\hline & Infiltr (1) & 2,011 & ,241 & 69,385 & 1 & ,000 & 7,473 \\
\hline & grado & & & 17,611 & $2(a)$ & ,000 & \\
\hline & grado (1) &, 775 & 1,030 &, 565 & 1 & ,452 & 2,170 \\
\hline & grado (2) & 1,977 & 1,023 & 3,735 & 1 & ,053 & 7,220 \\
\hline
\end{tabular}




\section{REFERENCIAS}

1. López-Abente G, Pollán M, Aragonés N, Pérez-Gómez B. Informe sobre la salud de los españoles. Cáncer. Informe de Área de Epidemiología Ambiental y Cáncer Centro Nacional de Epidemiología Instituto de Salud Carlos III. PDF [en linea] fecha de acceso 19 septiembre de 2005. URL disponible en http://cne.isciii.es/cancer.

2. Arrizabalaga M, Mora M, Navarro J, Extramiana J, Mañas A, Castro M, et al. Epidemiología descriptiva del cáncer vesical en el área sanitartia 8 de Madrid. Estudio retrospectivo sobre 315 pacientes. Actas Urol Esp. 1994;18(4):258-265.

3. Lynch CF, Cohen MB. Urinary system. Cancer. 1995;75(1 Suppl):316-329.

4. Moyano Calvo JL, Gutierrez Gonzalez M, Perez-Lanzac Llorca A, Molina Carranza A, Alvarez-Ossorio Fernandez JL, Sanchez Sanchez E, et al. Influencia de la edad en el comportamiento de los carcinomas uroteliales superficiales. Actas Urol Esp. 2005;29(1):82-88.

5. Madeb R, Messing EM. Gender, racial and age differences in bladder cancer incidence and mortality. Urol Oncol. 2004; 22(2):86-92.

6. Gardmark T, Bladstrom A, Hellsten S, Malmstrom PU, Members Of The Swedish Nacional Bladder Cancer Registry. Analysis of clinical characteristics, management and survival of patients with Ta T1 bladder tumours in Sweden between 1997 and 2001. Scand J Urol Nephrol. 2006;40(4): 276-282.

7. Moyano Calvo JL, Gutierrez Gonzalez M, Perez-Lanzac Llorca A, Molina Carranza A, Alvarez-Ossorio Fernandez JL, Sanchez Sanchez E, et al. Age's influence in superficial bladder cancer's behaviour. Actas Urol Esp. 2005;29(1):82-88.
8. Fine SW, Humphrey PA, Dehner LP, Amin MB, Epstein JI. Urothelial neoplasms in patients 20 years or younger: a clinicopathological analysis using the world health organization 2004 bladder consensus classification. J Urol. 2005; 174(5): 1976-1980.

9. Wen YC, Kuo JY, Chen KK, Lin AT, Chang YH, Hsu YS, et al. Urothelial carcinoma of the urinary bladder in young adults-clinical experience at Taipei Veterans General Hospital. J Chin Med Assoc. 2005;68(6):272-275.

10. Johnson DE, Hillis S. Carcinoma of the bladder in patients less than 40 years old. J Urol. 1978;120(2):172-173.

11. Wan J, Grossman B. Bladder carcinoma in patients age 40 years or younger. Cancer. 1989;64(1):178-181.

12. Lopez-Beltran A, Luque RJ, Alvarez-Kindelan J, Quintero A, Merlo F, Requena MJ, Montironi R. Prognostic factors in survival of patients with stage Ta and T1 bladder urothelial tumors: the role of G1-S modulators (p53, p21Waf1, p27Kip1, cyclin D1, and cyclin D3), proliferation index, and clinicopathologic parameters. Am J Clin Pathol. 2004; 122(3):444-452.

Correspondencia autora: Dra. P. Serrano Frago Servicio de Urología.

Hospital Universitario Miguel Servet

Po Isabel la Católica, 1-3. 50009 Zaragoza

Tel.: 976765500

e-mail autora: pserranof@comz.org

Información artículo: Original - Cáncer vesical

Trabajo recibido: octubre 2006

Trabajo aceptado: abril 2007 